Check for updates

Cite this: RSC Adv., 2017, 7, 34866

\title{
Polythiophene functionalized hydrophobic cellulose kitchen wipe sponge and cellulose fabric for effective oil-water separation $\uparrow$
}

\begin{abstract}
Nagarajan Durgadevi and Venkatanarasimhan Swarnalatha (iD *
Development of efficient materials for the separation of oils/organic solvents from water is of prime ecological importance as their negative impact on the aquatic environment is huge. In the present work, for the first time we report the utilization of biodegradable cellulose kitchen wipe sponge as a base material for the oil sorption and cellulose fabric as a filter for the filtration of oil, after functionalization with polythiophene. The water contact angles of the modified cellulose sponge and modified cellulose fabric being $126.6^{\circ}$ and $151.6^{\circ}$, respectively substantiate the hydrophobic nature of the materials post modification. Oil absorption kinetic studies show a very rapid saturation period (90 $\mathrm{min}$ ) for the modified cellulose sponge with a maximum absorption capacity of $7.5 \mathrm{~g} \mathrm{~g}^{-1}$. By a simple mechanical squeezing process, the absorbed oil/organic solvent is recycled. The sponge is reused for 5 cycles with $70 \%$ retention in the initial absorption efficiency. On the other hand, the modified cellulose fabric is used as a continuous filter for a quick separation of oil (and organic solvents) from water. The oil sorbents reported make use of readily available and economically viable base materials with a simple modification which may allow their use for the removal of oil on large scales.
\end{abstract}

Received 17th May 2017
Accepted 6th July 2017

DOI: 10.1039/c7ra05578a

rsc.li/rsc-advances

spilled oil in order to protect water and the lives dependent on water. Numerous techniques have been reported so far to clean up the spilled oil from the water surface, but the complete removal of the contaminants still remains intricate. General methodologies implemented for oil spill recovery are: (1) mechanical separation by the usage of booms, skimmers and sorbents. Booms and skimmers are used to collect the spilled oil and then separated mechanically; ${ }^{\mathbf{9} 10}$ (2) chemical separation method by the usage of solidifiers and dispersants. Solidifiers are high molecular weight polymers which get solidified when added to oil by elevating the viscosity of the oil. Dispersants are added to break the oil into small granules which can be further decomposed easily; ${ }^{11,12}$ (3) in situ burning of oil ${ }^{13}$ and (4) biological remediation using micro organisms like algae, bacteria, etc. The microbes transform the oil to comparatively less harmful substances via biodegradation mechanism. ${ }^{\mathbf{1 4 , 1 5}}$ Among these traditional methods, absorption is considered to be the most robust approach by far applied owing to its large adsorption capacity, high oil separation rate, cost-effectiveness, potential reusability, and ease to operate. Oil sorbents are functionalized materials which are capable of selectively absorbing either oil or water. Absorption materials which may necessitate extreme wettabilities such as superhydrophobicity, superhydrophilicity, superoleophobicity and superoleophilicity can be attained by modifying/functionalizing the chosen base materials. Absorbents utilized for the oil spill recovery until now can be extensively classified as (a) natural organic materials such as rice straw, silk fiber, kapok fiber, bagasse, wool fiber, corn 
stalks, natural rubber, ${ }^{16-22}$ (b) synthetic materials such as polypropylene, polyacrylate, alkyl acrylate copolymers, polyurethane, and polyolefin ${ }^{23-27}$ and (c) inorganic materials such as silica, zeolite, perlite, activated carbon and expanded graphite.,28-31 Materials belonging to each of these classes suffer from one or more drawbacks like complex synthetic strategy, difficulty to scale up, expenditure, lack of biodegradability and recyclability etc.

To function as an active oil/water separator, the materials selected should possess excellent hydrophobicity or oleophobicity. So, a readily available hydrophilic base material is often altered in order to increase hydrophobicity/oleophobicity. Recently, Zhu et al., worked on modifying the polyurethane sponges with electroless $\mathrm{Cu}$ deposition and further treating with $\mathrm{AgNO}_{3}$ and $n$-dodecanoic acid to impart hydrophobicity. ${ }^{32}$ Similarly, Zhou et al., fabricated superhydrophobic cotton fabrics by in situ vapor phase deposition of PANI and PTES for oil/water separation. ${ }^{33}$ Researchers also worked with carbon based materials viz. graphene treated melamine sponge, candle soot coated nickel foam and CNT/poly(dimethylsiloxane) coated polyurethane sponge. ${ }^{34-36}$ Even though several reported materials have been shown to function as exceptionally good oil water separators, their real time application is hampered due to the cost and complicated synthesis procedure. In this work, we aim to develop an inexpensive, easily scalable and hydrophobic/ oleophilic material with good selectivity towards oils from oilwater mixtures.

A well-established way to introduce hydrophobicity or superhydrophobicity to a surface of a material is through functionalizing via either silicon derivatives or fluorine containing compounds. ${ }^{18,36-38}$ In this research paper, neither silicon derivatives nor fluorinated compounds have been made use for achieving hydrophobicity. Polythiophene (PTh) has been selected as a compound to make a surface hydrophobic. Unlike these usual modifiers, here, we propose the incorporation of polythiophene (PTh) to a sponge wipe made up of natural cellulose that is utilized in kitchens. PTh, one of the most prominent conducting polymers and its derivatives have been used in a variety of applications such as batteries, field-effect transistors, photovoltaic devices and in biology. ${ }^{39-42}$ Pristine polythiophene is insoluble in many common solvents due to which it does not find scope in many areas. Due to the functionalities present in the polythiophene chain (aromatic ring containing a sulphur atom), it is hydrophobic. So far, there is no literature report available for demonstrating the hydrophobicity of polythiophene for environmental remediation or for any other application. Thus, the main objective of this work is the development of hydrophobic/ultrahydrophobic surfaces from hydrophilic cellulose materials via polythiophene for oil-water separation. The fabrication of polythiophene modified cellulose sponge and cellulose fabric is carried out through a facile single step synthesis by involving water as a solvent.

The cellulose sponge modified with PTh (PT-CS) is used as a sorbent and absorbs oil/organic solvent alone from the oilwater mixture. The cellulose fabric coated with PTh (PT-CF) is used as a filter and allows only the oil/organic solvent alone to pass through it.

\section{Experimental}

\section{Materials required}

Anhydrous ferric chloride and organic solvents were purchased from Rankem chemicals, India. Thiophene was procured from Alfa Aesar, India. All the chemicals purchased were of analytical grade and used as such without any further purification. Cellulose sponge $(20 \mathrm{~cm} \times 17.5 \mathrm{~cm})$ used as a kitchen wipe and cellulose fabric were purchased at local departmental stores. Oils used such as petrol (gasoline), diesel and motor oil were bought from local petrol stations. Kerosene and vacuum pump oil were purchased at local stores. Deionized water was utilized throughout the work.

\section{Polymerization of thiophene on the cellulosic sponge and fabric}

The surface of the cellulose sponge was functionalized through a facile single step oxidative polymerization of thiophene. Cellulose sponge was cut into small pieces with the dimensions of $2 \mathrm{~cm} \times 2 \mathrm{~cm} \times 0.35 \mathrm{~cm}$. The sponge was cleaned by immersing it in deionized water for 30 minutes to remove any soluble impurities, followed by drying in an oven at $70{ }^{\circ} \mathrm{C}$ for about 30 minutes. For $1.05 \mathrm{~g}(0.012 \mathrm{~mol})$ of thiophene, $8.9 \mathrm{~g}$ $(0.055 \mathrm{~mol})$ of anhydrous ferric chloride was used as a catalyst after its dissolution in $20 \mathrm{ml}$ of water..$^{43,44} 1 \mathrm{ml}$ thiophene was taken in a $100 \mathrm{ml}$ beaker in which the cellulose sponge was kept immersed for 30 minutes. Then the sponge was removed from thiophene and the total amount of thiophene absorbed by the sponge was assessed gravimetrically. This ensured the physisorption of thiophene on the sponge and aided to quantify the amount of catalyst to be added stoichiometrically. Besides, this step prevented the thiophene monomer getting clumsily polymerized in the reaction flask rather than on the sponge on the addition of a catalyst. The thiophene absorbed sponge was taken in a $250 \mathrm{ml} \mathrm{RB}$ flask and the required amount of ferric chloride solution was added to it. The reaction mixture was kept in an ice bath and the reaction was allowed to proceed for about $2 \mathrm{~h}$ under constant stirring. After $2 \mathrm{~h}$, the sponge was removed from the reaction mixture, washed repetitively well with deionized water and dried in a heating oven at $70{ }^{\circ} \mathrm{C}$ for 30 minutes.

The same procedure was adopted for the polymerization of thiophene on the surface of the cellulose fabric. Prior the polymerization, the cellulose fabric was cleaned well with acetone and deionized water to remove all the soluble impurities present in it.

\section{Characterization techniques}

The infrared spectra were recorded using Bruker Tensor 27 FTIR spectrometer scanning in the region ranging from 4000 to 600 $\mathrm{cm}^{-1}$ to confirm the presence of PTh after modification. The surface morphology of the cellulosic materials was analyzed using JEOL JSM - 6390LV scanning electron microscope at an accelerating voltage of $20 \mathrm{kV}$. The water contact angle (WCA) studies were done using KRUSS DSA E20 contact angle goniometer to measure the extent of hydrophobicity of the modified 
materials using the sessile droplet method. The mechanical properties of the modified and unmodified sponges were examined using Tinius Olson universal testing machine by the application of axial load until the material gets ruptured. Separation efficiency was cross-examined with thermogravimetric studies using Mettler Toledo TGA by heating the oil before and after filtration at a heating rate of $5{ }^{\circ} \mathrm{C}$ per minute under nitrogen atmosphere.

\section{Oil sorption studies}

Between the modified cellulose sponge and fabric, only the cellulose sponge with a large surface area was analyzed as oil absorbent. Oil absorption studies were carried out for the modified sponge by gravimetric method. The absorption of various types of oils (vacuum pump oil, motor oil, kerosene and petrol) and organic solvents (hexane and toluene) of different densities and viscosities were examined using the modified cellulose sponge. The pre-weighed sponge was kept immersed in oil/solvent. For every 10 minutes of the interval, the sponge was removed from oil, hung in the air for few seconds for oil to drip out and then weighed carefully to measure the weight of the absorbed oil/solvent. Extreme care was taken to avoid the evaporative loss of absorbed oil at room temperature. The kinetic studies were carried out until a saturation limit of oil/ solvent absorption was achieved. From the difference in the weights of the absorbent before and after oil absorption, the absorption capacity $(\kappa)$ of the sponge was calculated using the relation,

$$
\kappa=\frac{W_{1}-W_{0}}{W_{0}},
$$

where $W_{1}$ and $W_{0}$ are the weights of the sponge after and before the sorption process, respectively.

\section{Effect of temperature and ionic strength}

Influence of temperature and salinity (to understand the real possible application in the marine environment) were investigated by using high density motor oil. High density motor oil was selected as example oil as it had the highest absorption capacity exhibited by the modified sponge. The percentage absorption of PT-CS sponge was calculated after 1 hour immersion in oil, maintained under various temperature conditions ranging from $0{ }^{\circ} \mathrm{C}$ to $50{ }^{\circ} \mathrm{C}$. And the effect of ionic strength was studied by choosing the common salt sodium chloride as an electrolyte. The PT-CS sponge was immersed for 1 hour in a mixture containing sodium chloride and motor oil and then the absorption capacities were calculated. Saline solutions of various concentrations ranging from $0.1 \mathrm{M}$ to $0.5 \mathrm{M}$ were utilized for studies and the corresponding percentage absorption of motor oil was determined.

\section{Recyclability studies}

The spilled oil/solvent that was absorbed by the sponge could be reused again after recovering it via a simple mechanical squeezing process. After the mechanical squeezing, to make sure the complete removal of the absorbed oil/solvent, the sponge was washed with hexane and dried in a hot air oven at $70{ }^{\circ} \mathrm{C}$ for 30 minutes. After the PT-CS sponge had been allowed to cool down, it was reused for the next cycle of oil absorption. The absorption efficiencies for 5 cycles were recorded.

\section{Oil-water separation efficiency}

The superhydrophobic cotton fabric prepared is analyzed for its efficacy as continuous oil-water separator. Gravity driven separation methodology using an ordinary funnel and a round bottom flask was followed for the calculation of separation efficiencies. To demonstrate the oil-water separation via the fabricated filter, the PT-CF fabric was first placed inside a funnel. A mixture containing equal amounts of oil and water was poured into the funnel. Extreme care was taken during the volume measurement by keeping in mind the volatile nature of few of the oils involved in the study. The oil-water separation efficiency (SE) was evaluated using the formula. ${ }^{45}$

$$
\mathrm{SE}=\frac{V_{\text {iso }}+V_{\mathrm{s}}}{V_{0}} \times 100,
$$

where $V_{\text {iso }}$ corresponds to the volume of the isolated oil after filtration and $V_{\mathrm{s}}$ is the volume of oil slightly absorbed by the fabric, respectively. The volume of oil before the filtration process is denoted by $V_{0}$.

\section{Results and discussion}

The formation of PTh on the cellulose materials is verified by FTIR spectral data. The IR spectra for the modified and unmodified cellulose fabric are shown in Fig. 1. The raw cellulose fabric shows the usual IR pattern which has characteristic peaks of the functional groups present in cellulose. The peak present at $1642 \mathrm{~cm}^{-1}$ is due to water absorbed by cellulose. ${ }^{46}$ The cellulose fabric after the modification gives rise to significant peaks authenticating the formation of PTh on the surface of the material. The decrease in the value of transmittance postmodification might be due to the formation of a denser and

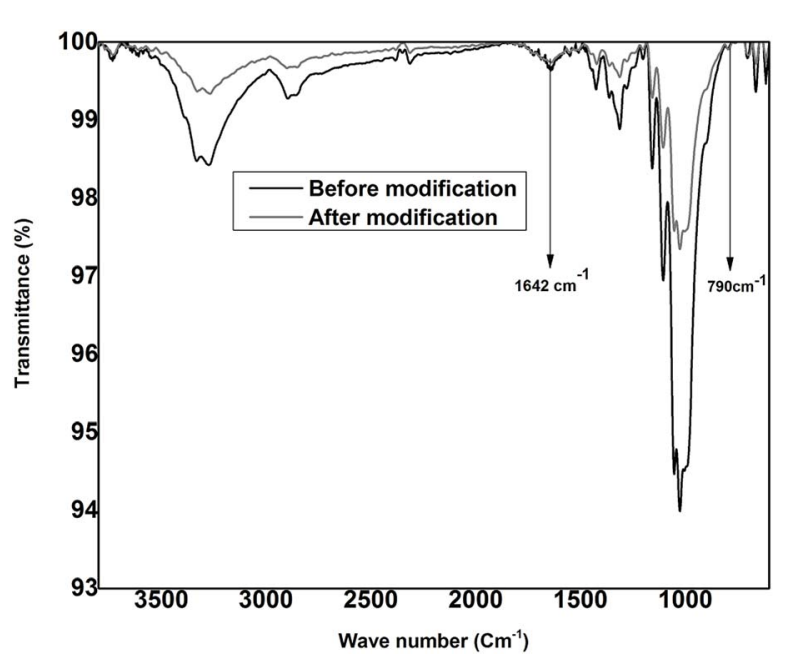

Fig. 1 FTIR spectra of unmodified cellulose fabric and modified cellulose fabric with polythiophene. 
smoother PTh layer on the surface of the material. The new peak appeared around $790 \mathrm{~cm}^{-1}$ can be associated with 2,5-disubstituted thiophene, which confirms the presence of thiophene in its polymeric form. ${ }^{47}$ The peak shown at $1642 \mathrm{~cm}^{-1}$ due to the absorbed water is present in the spectra of both unmodified and modified fabric. A peak at the same region can be accounted for a stretching vibration of $-\mathrm{C}=\mathrm{C}$ bonds. But it can be clearly seen that the intensity and sharpness of the peak have been enhanced to a greater degree after the polymerization. The reason for this deviation is that there would be an additional contribution from polythiophene $-\mathrm{C}=\mathrm{C}$ bonds after the polymerization.

Surface morphological studies and elemental analysis of the cellulosic material before and after the modification are done via SEM imaging technique. The microscopic images and the energy dispersive X-ray spectroscopic (EDAX) images are given in Fig. 2. From the SEM image of the modified cellulose surface, it is highly evident that the PTh develops uniform coating over the cellulose surface, thereby smoothening its surface in comparison with that of raw cellulose. Also, the SEM image of the unmodified fabric possesses a bright lining at the edges of the fibers as a result of the charge accumulation or building up of static electrical charges on top of the specimen. This bright line seems to be missing in the PTh modified material whose SEM analysis was also recorded under identical parameters and conditions. During SEM image acquisition, the charging effect on the surface can be typically minimized by coating with a conductive material. Therefore, the reduction in the charging effect must be due to the presence of a thin conductive film which is polythiophene in this study. This observation as well ascertains the formation of PTh on the surface of the material.
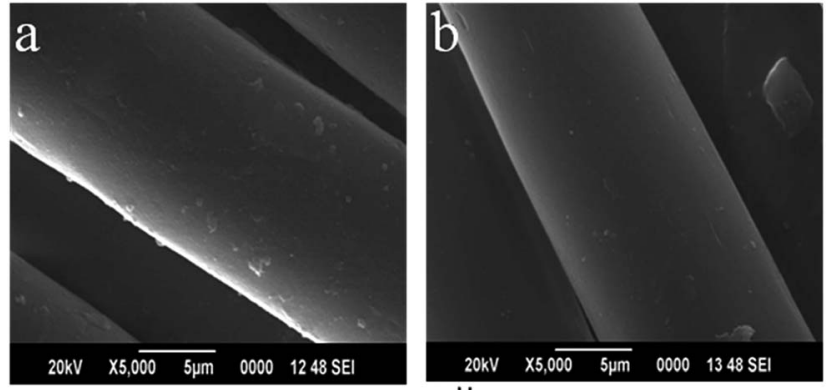

C

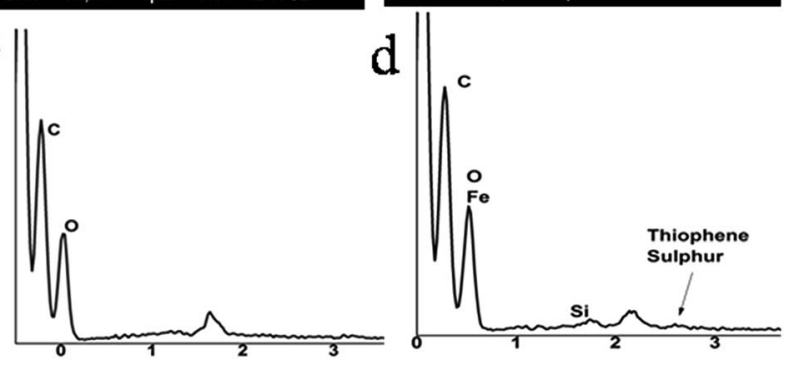

Fig. 2 SEM and EDAX spectra of the cellulose material. (a) The SEM image of the unmodified material showing the charge accumulation on the surface. (b) The SEM image of the PT-CF showing the uniform coating of PTh (c) EDAX spectra of the unmodified material (d) EDAX spectra of the PT-CF showing the characteristic presence of sulphur at $2.6 \mathrm{keV}$.
Along with SEM images, the corresponding EDAX spectra are also recorded and the data are shown in Fig. $2 \mathrm{c}$ and d. In EDAX spectra, the K $\alpha$ emission band for elemental sulphur is found at $2.3 \mathrm{keV}$. In contrast, a shift in the energy of the X-rays leads to an identical peak appearing at $2.6 \mathrm{keV}$, which is a distinctive peak obtained for thiophenic sulphur. ${ }^{48}$ The EDAX result presented in Fig. 2d agrees well with this literature data and supports the formation of polythiophene under the normal reaction conditions followed in this work. The composition data obtained reveals the quantity of sulphur loaded to the cellulose material is very low.

Wettability of the modified and unmodified materials is explored by static water contact angle measurements. When a drop of water is added onto the surface of the raw cellulose sponge, it absorbs the water droplet immediately as the contact angle being nearly zero. The same behavior is witnessed on the surface of the fabric too. On the contrary, water remains on the surface without penetrating deeper, when placed on top of the modified sponge and the modified fabric. The hydrophobic nature of the cellulose materials after the modification has been given in Fig. 3. The modified fabric exhibits a water contact angle around $151.6^{\circ}$ making it an ultrahydrophobic material as shown in Fig. 3d. On the other hand, the modified sponge displays a water contact angle of $126.6^{\circ}$. The results imply that the superhydrophobic polythiophene film follows Cassie-Baxter model. ${ }^{49,50}$ The model suggests that on a hydrophobic body, a liquid droplet remains floating on the surface, creating solidair-liquid interface which in turn acts as the cushion. ${ }^{51}$ The dissimilarities in the extents of wettability is owing to the fact that the surface of the fabric is more uniform as against the relatively rough surface present in the cellulose sponge. This disparity in the nature of the surface may result in different extents of polymerization and functionalities. These are one of

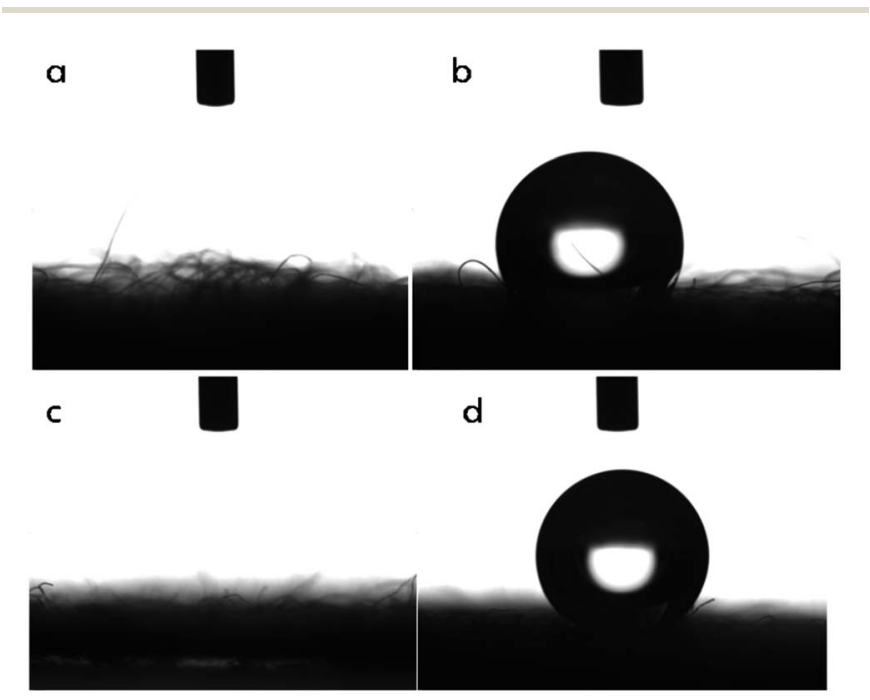

Fig. 3 Images used for contact angle measurements (a) the zero contact angle for the cellulose sponge on the addition of a water droplet. (b) PT-CS with contact angle of about $126.6^{\circ}$ on the addition of a water droplet. (c) The zero contact angle for the cellulose fabric on the addition of a water droplet. (d) PT-CF with contact angle of about $151.6^{\circ}$ on the addition of a water droplet. 
the most important factors which determine the surface free energies. The wettability experiments illustrate that the modified sponge is hydrophobic and the modified fabric is ultrahydrophobic in nature, thus making the polythiophene coated fabric an ideal material for oil-water filtration and the polythiophene coated sponge for oil absorption.

The photographic images of the modified fabric showing the hydrophobic character are given in Fig. 4. Water gets absorbed by the fabric instantly (Fig. 4a) but remains on the surface of the polythiophene modified fabric (Fig. 4b). The same effect is with the PT-CS sponge and also it exhibits good buoyancy property on the surface of the water shown in Fig. 4c.

From the above results, it is clear that the material exhibits hydrophobic behavior and further PT-CS sponge can be used as the sorbent material for the separation of oil from water. Absorption capability and the duration for the corresponding oil absorption determine whether a synthesized material would be an effective absorbent or not. From the percentage absorption values calculated, we understand that an oil/solvent with high density gets absorbed to a greater extent when compared to that with low density, by the modified cellulose sponge. The density values for the various oils/solvents used are provided in Table 1. Among the oils used, motor oil having the highest density $\left(0.8503 \mathrm{~g} \mathrm{ml}^{-1}\right)$ shows a percentage absorption of 748 , which is the highest \% absorption exhibited by the sponge. It is reported that the oil with high viscosity will adhere strongly to a hydrophobic material via intermolecular hydrophobic interaction and thereby exhibits the high oil absorption capacity. ${ }^{\mathbf{1 6}}$ The supposition is confirmed by the absorption behavior shown by toluene. Even though the density of toluene is equally as high as that of motor oil $\left(0.863 \mathrm{~g} \mathrm{ml}^{-1}\right)$, the percentage absorption is around 570 due to the relatively lower viscosity of toluene. $1 \mathrm{~g}$ of PT-CS sponge absorbs $7.5 \mathrm{~g}$ of the high density motor oil and reaches the saturation limit promptly in 60 minutes. The PT-CS sponge attains saturation within 90 minutes towards all the oils/solvents taken for the study, irrespective of their viscosities. A kinetics graph is plotted between time and percentage absorption as shown in Fig. 5. The kinetic plot affirms that the absorption phenomenon follows Fickian diffusion mechanism.

To expand the applicability of the proposed materials from a lab to a marine ecosystem, it is essential to understand the oil uptake behaviour at different temperatures and different degrees of salinities. There is a small change in the magnitude of the absorption capacities while swinging the temperature

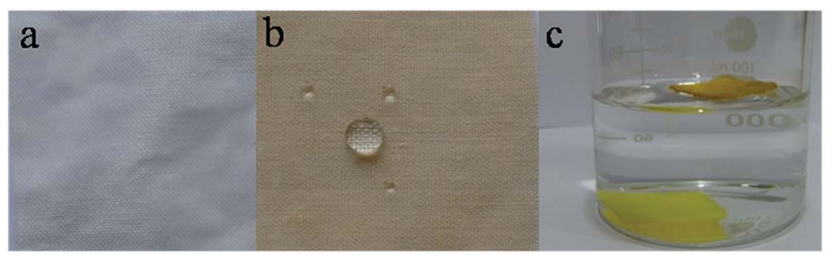

Fig. 4 (a) Unmodified cellulose fabric absorbs the water. (b) The modified PT-CF shows the rolling water droplet on the surface. (c) The modified PT-CS sponge shows the buoyancy property on the water surface whereas the unmodified cellulose sponge sinks within seconds.
Table 1 Oils/solvent used along with the density and absorption maximum

\begin{tabular}{llll}
\hline S. no & Oil/solvent & $\begin{array}{l}\text { Density } \\
\left(\mathrm{g} \mathrm{ml}^{-1}\right)\end{array}$ & $\begin{array}{l}\text { Maximum \% } \\
\text { absorption }\end{array}$ \\
\hline 1 & Hexane & 0.655 & 433 \\
2 & Toluene & 0.863 & 573 \\
3 & Petrol & 0.7139 & 442 \\
4 & Kerosene & 0.7938 & 498 \\
5 & Vacuum pump oil & 0.8196 & 633 \\
6 & Motor oil & 0.8503 & 748 \\
\hline
\end{tabular}

from $0{ }^{\circ} \mathrm{C}$ to $50{ }^{\circ} \mathrm{C}$. The absorption capacity of the sponge varies from $7.6 \mathrm{~g} \mathrm{~g}^{-1}$ to $6.8 \mathrm{~g} \mathrm{~g}^{-1}$ on increasing the temperature from $0{ }^{\circ} \mathrm{C}$ to $50{ }^{\circ} \mathrm{C}$ (Fig. 6a). As the temperature parameter has a very profound effect on viscosity, with reduction in temperature, the viscosity of the oil increases and the reverse occurs with increase in temperature. As discussed earlier viscosity plays a vital role in oil absorption capacity and hence the PT-CS sponge exhibits higher absorption at low temperature than higher temperature.

The PT-CS sponge remains stable on increasing the ionic strength of the water from $0.1 \mathrm{M}$ to $0.5 \mathrm{M}$ (Fig. 6b). The increase in salinity is found to not affect the absorption capabilities as well as the buoyancy of the PT-CS sponge. These results authenticate the usage of the cellulose sponge in sea water.

For a practically exploitable reusable oil absorbent material, mechanical properties such as tensile strength, extension and elasticity are imperative characteristics. Therefore, a tensile stress-strain percentage curve is drawn to analyze the tensile strength and the shape retention capability of the material (Fig. 7a). Both the modified and unmodified cellulose sponges show a non-linear elastic behavior at the beginning load range and after that displays a constant strain before getting ruptured. This trend variation in the strain might be due to the

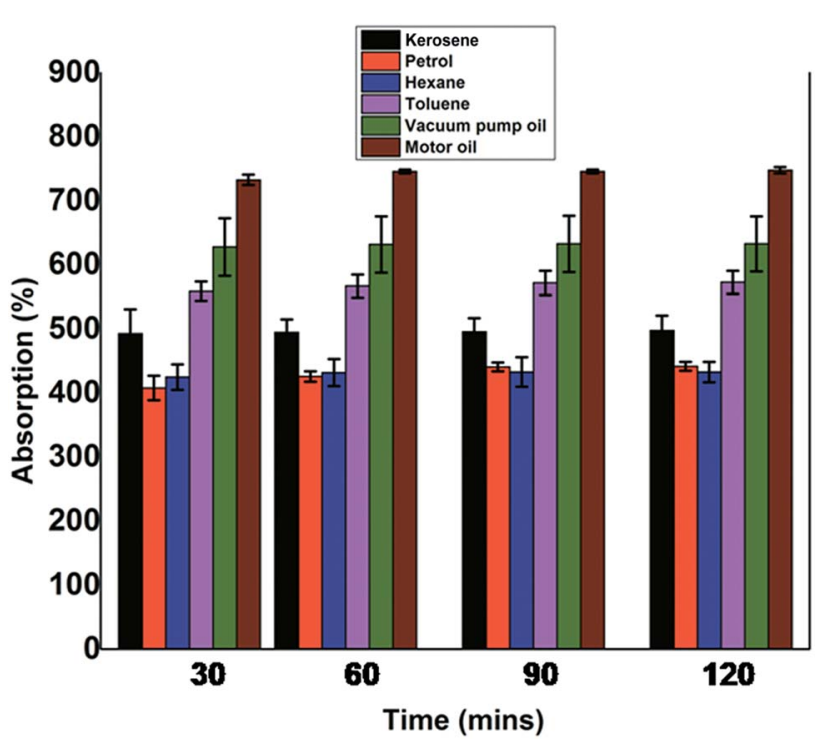

Fig. 5 Oil/solvent absorption and kinetic studies. The PT-CS sponge shows maximum absorption and attains saturation within 90 min. 

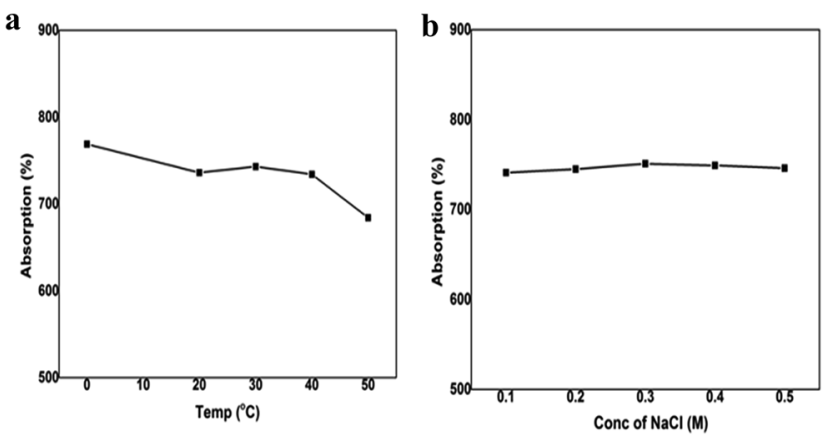

Fig. 6 (a) Plot between percentage absorption versus temperature for motor oil; there is a decrease in the absorption capacity on increasing the temperature to $50{ }^{\circ} \mathrm{C}$ (b) plot between percentage absorption versus concentration of sodium chloride showing the stability of the PT-CS in $\mathrm{NaCl}$ medium.

orientation of the cellulose fibers being random and uniform with respect to the direction of the tensile stress applied, at lower and higher loads respectively. It is also seen that the percentage of strain at which the breaking occurs decreases from $31 \%$ to $17.5 \%$ after the modification. The tensile strength values for the unmodified and modified cellulose sponge are 2.28 MPa and 1.87 MPa, respectively. From these inferences, it is learnt that introduction of polythiophene on the cellulose sponge has caused a profound variation in the viscoelastic properties of the material. This observation can be rationalized as the diminishing of the extensive intermolecular interactions present in the raw cellulose occurs due to the presence of rigid polythiophene units. Additionally, a load versus extension graph is presented in Fig. $7 \mathrm{~b}$. The raw cellulose sponge acquires the maximum load of $50 \mathrm{~N}$ whereas the modified material reaches the maximum elongation by applying the load of $41 \mathrm{~N}$ itself. These studies reveal that the mechanical properties have drastically changed after the modification process. Both the sponges possess very good shape retention ability i.e. the materials regain their shape and size even after the application and release of a very high load. The mechanical studies were done at ambient temperature conditions.
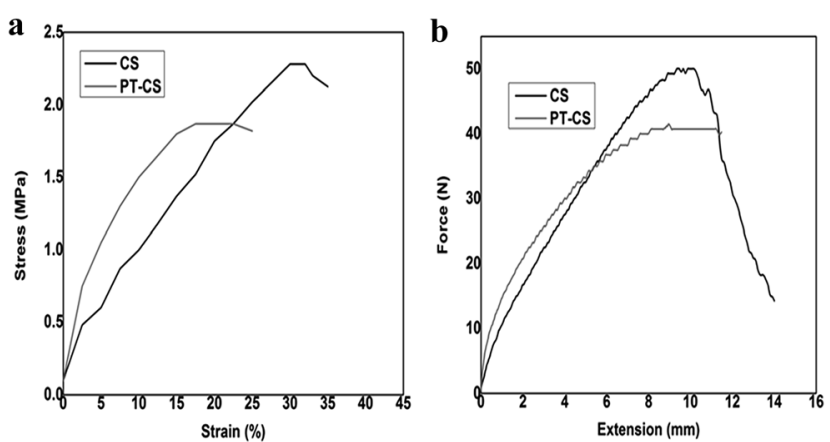

Fig. 7 (a) The stress-strain plot of the modified and unmodified cellulose sponge. (b) The plot between the applied force and the corresponding extension of the modified and unmodified cellulose sponge.

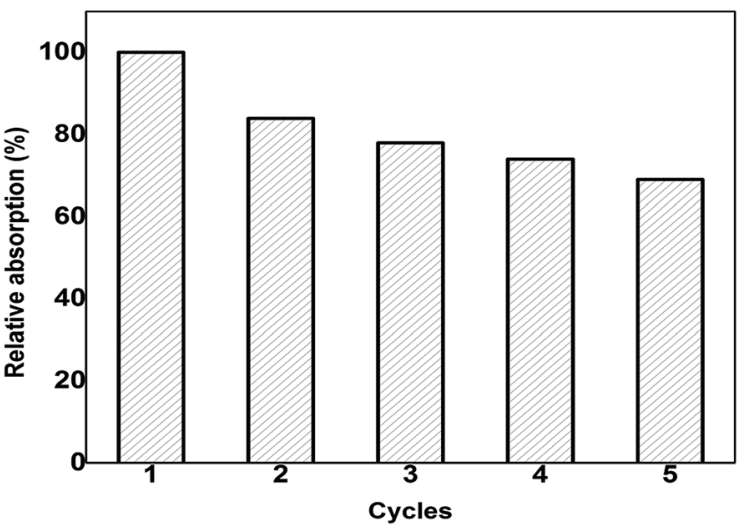

Fig. 8 Graph between percentage absorption and number of cycles; there is $30 \%$ loss in the absorption capacity on reusing the sponge for the 5 th cycle.

For a successful absorbent material, reusability is the desirable quality due to which the preparation cost and time can be minimized. From recyclability studies, it is determined that the PT-CS sponge can be reused for 5 cycles with the retention of $70 \%$ of the original absorption efficiency. The oil/ solvent is removed from the sponge by a simple mechanical squeezing process and the oil isolated can be reused. On increasing the number of cycles the absorption capacity of the sponge is found to diminish gradually and a graph is plotted between percentage absorption and number of cycles (Fig. 8).

When a mixture containing oil and water is poured through the modified fabric, the oil alone starts draining through the stem of the funnel as the fabric restricts the passage of water through it (Fig. 9). Thus driven by gravitational force, the oil gets separated out and is collected in a container within few seconds shown in the Video (ESI $†$ ). For the identification purpose, water is dyed with rhodamine B and oil/solvent is used as such. By this simple procedure, a large quantity of oil/solvent contaminated with

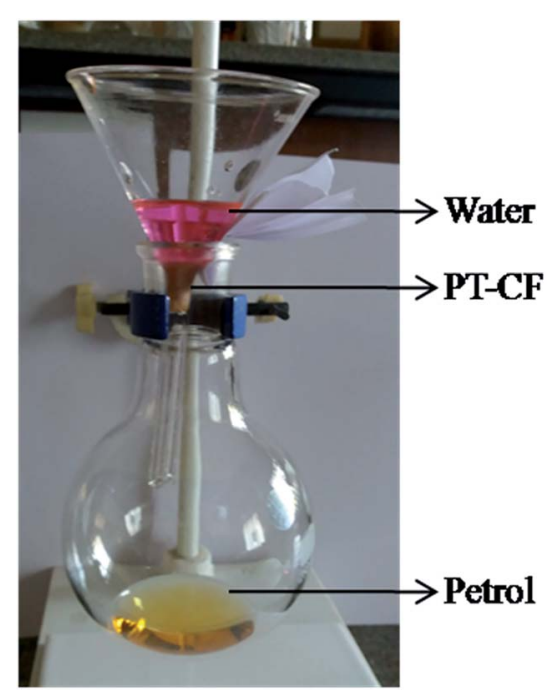

Fig. 9 Filtration of the petrol-water mixture using modified fabric with the help of simple funnel and round bottom flask setup. 


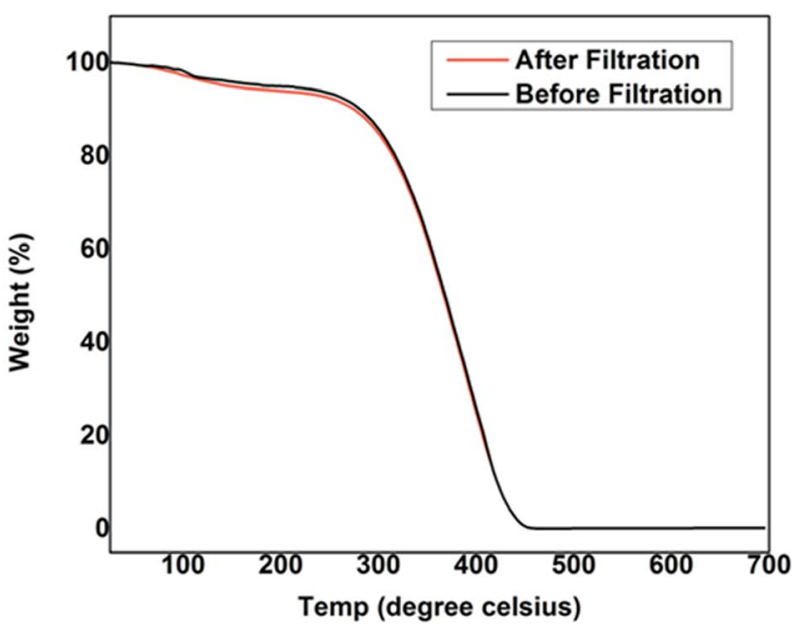

Fig. 10 The TGA plot of the vacuum pump oil taken before and after filtration using PT-CF showing $99.2 \%$ separation efficiency.

water can be separated easily and rapidly. The separation efficiency values are found to vary a little bit with respect to the nature of the oils, where the average separation efficiency is found to be $99.2 \%$ for the modified cotton fabric. To crossexamine the results acquired using volume data, we tried to repeat analyzing the separation efficiency with thermogravimetric analysis. As a result of high volatility associated with few oils, we could not perform the TGA for all the oils. For TGA trial, vacuum pump oil with relatively less volatility is chosen. The thermal analyses of the vacuum pump oil before and after filtration are done as shown in Fig. 10. The result interpreted from the thermogram i.e. the difference in the weights of the oil before and after filtration matches with the results received earlier from the volume data, which is $99.2 \%$. This assures the plausible utility of the modified cotton fabric as oil water separator.

\section{Conclusion}

A simple, inexpensive and easily scalable hydrophobic material is proposed for oil-water separation. Biodegradable cellulose sponge and cellulose fabric are taken as base materials and they are treated with polythiophene. The PT-CF fabric shows superhydrophobic behavior with the water contact angle around $151.6^{\circ}$ and the PT-CS have the water contact angle around $126.6^{\circ}$. The PT-CS sponge acts as good oil absorbent and the PT$\mathrm{CF}$ fabric acts as a good filter to separate oil and water. The PTCS sponge withstands both high and low temperature and also remains stable on increasing the salinity of the water. Mechanical studies also authenticate the durability of the sponge as effective oil absorbent. One gram of the PT-CS sponge absorbs a maximum of $7.5 \mathrm{~g}$ of high density motor oil. Both the PT-CS sponge and the PT-CF fabric can be reused for several cycles without much loss in the absorption property. Thus, the above prepared material promises to be a potential adsorbent for large scale separation of oil-water as well as for small scale cleanings in labs. The precursor materials chosen are costeffective and safe to handle leading to a feasible utility of the proposed materials commercially.

\section{Acknowledgements}

The authors thank Dr R. T. Rajendra Kumar, Dept of Nanoscience and Technology, Bharathiar University, India for water contact angle measurements.

\section{References}

1 Information for action [Internet], 2015, http:// www.informaction.org/index.php?

main $=$ waterpol_gen\&subject $=$ Water\%20sewage.

2 S. T. Nguyen, J. Feng, N. T. Le, A. T. T. Le, N. Hoang, V. B. C. Tan, et al., Cellulose aerogel from paper waste for crude oil spill cleaning, Ind. Eng. Chem. Res., 2013, 52(51), 18386-18391.

3 L. Yu, G. Hao, Q. Liang and W. Jiang, Fabrication of magnetic porous silica submicroparticles for oil removal from water, Ind. Eng. Chem. Res., 2015, 54(38), 9440-9449.

4 N. Zhang, W. Jiang, T. Wang, J. Gu, S. Zhong, S. Zhou, et al., Facile preparation of magnetic poly(styrene-divinylbenzene) foam and its application as an oil absorbent, Ind. Eng. Chem. Res., 2015, 54(44), 11033-11039.

5 Y. Yang, M. Hu, D. Zhou, W. Fan, X. Wang and M. Huo, Bioremoval of $\mathrm{Cu}^{2+}$ from CMP wastewater by a novel copper-resistant bacterium Cupriavidus gilardii CR3: characteristics and mechanisms, RSC Adv., 2017, 7(30), 18793-18802.

6 Y.-Q. Li, Y. A. Samad, K. Polychronopoulou, S. M. Alhassan and K. Liao, Carbon aerogel from winter melon for highly efficient and recyclable oils and organic solvents absorption, ACS Sustainable Chem. Eng., 2014, 2(6), 1492-1497.

7 A. Taylor, The Exxon Valdez oil spill: 25 years ago today, 2014, https:/www.theatlantic.com/photo/2014/03/the-exxonvaldez-oil-spill-25-years-ago-today/100703/\#article.

8 P. Kumar, How North Chennai's Kamarajar port made a bad oil spill worse, 2017, https:www.//thewire.in/105986/oil-spillennore-kamarajar/.

9 F. A. Richards, Oil skimmer, Grant US3700108 A, 1972.

10 Q. F. Wei, R. R. Mather, A. F. Fotheringham and R. D. Yang, Evaluation of nonwoven polypropylene oil sorbents in marine oil-spill recovery, Mar. Pollut. Bull., 2003, 46(6), 780-783.

11 S. R. Jadhav, P. K. Vemula, R. Kumar, S. R. Raghavan and G. John, Sugar-derived phase-selective molecular gelators as model solidifiers for oil spills, Angew. Chem., Int. Ed., 2010, 122(42), 7861-7864.

12 R. R. Lessard and G. DeMarco, The Significance of Oil Spill Dispersants, Spill Sci. Technol. Bull., 2000, 6(1), 59-68.

$13 \mathrm{~J}$. V. Mullin and M. A. Champ, Introduction/overview to in situ burning of oil spills, Spill Sci. Technol. Bull., 2003, 8(4), 323-330.

14 I. M. Banat, Biosurfactants production and possible uses in microbial enhanced oil recovery and oil pollution remediation: a review, Bioresour. Technol., 1995, 51(1), 1-12.

15 R. Balachandar, M. Jayakumar, A. Thangaraja, B. Bharathiraja, P. Gurumoorthy and S. Bharathy, Use of 
luminous bacteria as a biosensor for monitoring of heavy metals in marine water, Int. J. Chem. Sci., 2010, 8, 1553-1566.

16 M. Patowary, K. Pathak and R. Ananthakrishnan, Robust superhydrophobic and oleophilic silk fibers for selective removal of oil from water surfaces, $R S C A d v$., 2016, 6(77), 73660-73667.

17 X.-F. Sun, R. C. Sun and J.-X. Sun, Acetylation of rice straw with or without catalysts and its characterization as a natural sorbent in oil spill cleanup, J. Agric. Food Chem., 2002, 50(22), 6428-6433.

18 J. Wang, G. Geng, X. Liu, F. Han and J. Xu, Magnetically superhydrophobic kapok fiber for selective sorption and continuous separation of oil from water, Chem. Eng. Res. Des., 2016, 115, 122-130.

19 G. G. Monica Periolatto, Greasy raw wool for clean-up process of marine oil spill: from laboratory test to scaled prototype, Chem. Eng. Trans., 2015, 43, 2269-2274.

20 R. Behnood, B. Anvaripour, N. Jaafarzadeh and M. Farasati, Oil spill sorption using raw and acetylated sugarcane bagasse, J. Cent. South Univ., 2016, 23(7), 1618-1625.

$21 \mathrm{M}$. Husseien, A. A. Amer, A. El-Maghraby and N. Hamedallah, Oil spill removal from water by using corn stalk: factors affecting sorption process, Int. J. Environ. Waste Manage., 2015, 16(4), 281-292.

22 S. Venkatanarasimhan and D. Raghavachari, Epoxidized natural rubber-magnetite nanocomposites for oil spill recovery, J. Mater. Chem. A, 2013, 1(3), 868-876.

$23 \mathrm{~J}$. Zhao, C. Xiao and N. Xu, Evaluation of polypropylene and poly(butylmethacrylate-co-hydroxyethylmethacrylate) nonwoven material as oil absorbent, Environ. Sci. Pollut. Res., 2013, 20(6), 4137-4145.

24 J. Jang and B.-S. Kim, Studies of crosslinked styrene-alkyl acrylate copolymers for oil absorbency application. I. Synthesis and characterization, J. Appl. Polym. Sci., 2000, 77(4), 903-913.

25 J. S. Yang, S. M. Cho, B. K. Kim and M. Narkis, Structured polyurethanes for oil uptake, J. Appl. Polym. Sci., 2005, 98(5), 2080-2087.

26 P. Fang, P. Mao, J. Chen, Y. Du and X. Hou, Synthesis and properties of a ternary polyacrylate copolymer resin for the absorption of oil spills, J. Appl. Polym. Sci., 2014, 131(8), 40180.

27 X. Yuan and T. C. M. Chung, Novel solution to oil spill recovery: using thermodegradable polyolefin oil superabsorbent polymer (oil-SAP), Energy Fuels, 2012, 26(8), 4896-4902.

28 T. Sakthivel, D. L. Reid, I. Goldstein, L. Hench and S. Seal, Hydrophobic high surface area zeolites derived from fly ash for oil spill remediation, Environ. Sci. Technol., 2013, 47(11), 5843-5850.

29 D. Bastani, A. A. Safekordi, A. Alihosseini and V. Taghikhani, Study of oil sorption by expanded perlite at $298.15 \mathrm{~K}$, Sep. Purif. Technol., 2006, 52(2), 295-300.

30 A. L. Ahmad, S. Sumathi and B. H. Hameed, Residual oil and suspended solid removal using natural adsorbents chitosan, bentonite and activated carbon: a comparative study, Chem. Eng. J., 2005, 108(1-2), 179-185.
31 T. Yao, Y. Zhang, Y. Xiao, P. Zhao, L. Guo, H. Yang, et al., The effect of environmental factors on the adsorption of lubricating oil onto expanded graphite, J. Mol. Liq., 2016, 218, 611-614.

32 Q. Zhu, Q. Pan and F. Liu, Facile removal and collection of oils from water surfaces through superhydrophobic and superoleophilic sponges, J. Phys. Chem. C, 2011, 115(35), 17464-17470.

33 X. Zhou, Z. Zhang, X. Xu, F. Guo, X. Zhu, X. Men, et al., Robust and durable superhydrophobic cotton fabrics for oil/water separation, ACS Appl. Mater. Interfaces, 2013, 5(15), 7208-7214.

34 F. Zhao, L. Liu, F. Ma and L. Liu, Candle soot coated nickel foam for facile water and oil mixture separation, $R S C A d v$, 2014, 4(14), 7132-7135.

35 J. Zhao, Q. Guo, X. Wang, H. Xie and Y. Chen, Recycle and reusable melamine sponge coated by graphene for highly efficient oil-absorption, Colloids Surf., A, 2016, 488, 93-99.

36 C.-F. Wang and S.-J. Lin, Robust superhydrophobic/ superoleophilic sponge for effective continuous absorption and expulsion of oil pollutants from water, ACS Appl. Mater. Interfaces, 2013, 5(18), 8861-8864.

37 C. Xuemei, A. W. Justin and V. G. Suresh, Continuous oilwater separation using polydimethylsiloxanefunctionalized melamine sponge, Ind. Eng. Chem. Res., 2016, 55(12), 3596-3602.

38 R. Li, C. Chen, J. Li, L. Xu, G. Xiao and D. Yan, A facile approach to superhydrophobic and superoleophilic graphene/polymer aerogels, J. Mater. Chem. A, 2014, 2(9), 3057-3064.

39 C. Y. Wang, A. M. Ballantyne, S. B. Hall, C. O. Too, D. L. Officer and G. G. Wallace, Functionalized polythiophene-coated textile: a new anode material for a flexible battery, J. Power Sources, 2006, 156(2), 610-614.

40 H. Koezuka, A. Tsumura and T. Ando, Field-effect transistor with polythiophene thin film, Synth. Met., 1987, 18(1), 699704.

$41 \mathrm{~J}$. Chen and Y. Cao, Development of novel conjugated donor polymers for high-efficiency bulk-heterojunction photovoltaic devices, Acc. Chem. Res., 2009, 42(11), 17091718.

42 S. Das, D. P. Chatterjee, R. Ghosh and A. K. Nandi, Water soluble polythiophenes: preparation and applications, $R S C$ Adv., 2015, 5(26), 20160-20177.

43 R. Liu and L. Z. Chin, Polythiophene: synthesis in aqueous medium and controllable morphology, Chin. Sci. Bull., 2009, 54, 2028-2032.

44 A. Gök, M. Omastová and A. G. Yavuz, Synthesis and characterization of polythiophenes prepared in the presence of surfactants, Synth. Met., 2007, 157(1), 23-29.

45 C. R. Reshmi, P. S. Suja, A. Juraij and A. Sujith, Fabrication of superhydrophobic polycaprolactone/beeswax electrospun membranes for high efficiency oil/water separation, $R S C$ Adv., 2017, 7, 2092-2102.

$46 \mathrm{~J}$. Lojewskaa, P. Miśkowieca, T. Qojewskia and L. M. Proniewicz, Cellulose oxidative and hydrolytic 
degradation: in situ FTIR approach, Polym. Degrad. Stab., 2005, 88(3), 512-520.

47 O. Inganäs, B. Liedberg, W. Chang-Ru and H. Wynberg, A new route to polythiophene and copolymers of thiophene and pyrrole, Synth. Met., 1985, 11(4), 239-249.

48 G. P. Huffman, F. E. Huggins, S. Mitra, N. Shah, R. J. Pugmire, B. Davis, et al., Investigation of the molecular structure of organic sulfur in coal by XAFS spectroscopy, Energy Fuels, 1989, 3(2), 200-205.
49 R. N. Wenzel, Resistance of Solid Surfaces to Wetting by Water, Ind. Eng. Chem., 1936, 28(8), 988-994.

50 A. B. D. Cassie and S. Baxter, Wettability of porous surfaces, Trans. Faraday Soc., 1944, 40, 546-551.

51 J. C. Palacios, G. J. Cruz, M. G. Olayo and J. A. ChávezCarvayar, Characterization of hydrophobic and hydrophilic polythiophene-silver-copper thin film composites synthesized by DC glow discharges, Surf. Coat. Technol., 2009, 203(21), 3032-3036. 